\title{
ON ARITHMETICAL CLASSES NOT CLOSED UNDER DIRECT UNION ${ }^{1}$
}

KURT BING

1. Introduction. Horn [2, Theorems 4 and 10; cf. 3] has shown that every class of algebras characterizable by a closed sentence of conditional type is closed under passage to direct union and that this result cannot be improved by allowing a larger class of characterizing sentences describable in terms of the quantifiers and propositional structure of their prenex normal forms. It is not known whether every class of algebras which is characterizable by a closed sentence, but not by a closed sentence of conditional type, fails to be closed under direct union.

These results and problems carry over into Tarski's theory of arithmetical classes of algebras [4], ${ }^{2}$ which provides mathematical counterparts for characterizing sentences containing relation symbols, rather than for the more special sentences containing operation symbols used by Horn [2]. In particular, it is known that all conditional classes of algebras, as defined below, are closed under direct union, ${ }^{2}$ and the problem whether all other (nonconditional) arithmetical classes of algebras fail to be closed under direct union is still unsolved. However, we shall give two sufficient conditions for nonclosure under direct union of nonconditional classes ( $\$ 2$, Theorems 1 and 2), as well as a sufficient condition for closure, of which we do not know that it applies to conditional classes only ( $\$ 3$, Theorem 3 ). These results will be further discussed in $\$ 3$.

We use the terminology and notation of [4], with the provision that + shall be considered as a ternary relation (not as a binary operation), and the necessary changes be made in subsequent definitions. This restriction to one finitary relation is not essential for the ideas used. For purposes of abbreviation, we use the symbols $\wedge$ (and), $\rightarrow$ (if $\cdots$ then $\cdots$.,$\vee$ (there exists), $\wedge$ (for all), and such symbols as $\bigvee i \in I$ (there exists an element $i$ of $I$ ) and $\wedge i \in I$ (for all elements $i$ of $I$ ).

Presented to the Society, October 30,1954; received by the editors November 10, 1954.

1 The problem considered in this paper was suggested to the author by Professor Alfred Tarski in his seminar of Spring 1953 at the University of California. Professor Tarski also suggested Corollary 1 of this paper and the use of a method due to McKinsey [1, Theorem 1] for its proof. This method appears in the proofs of Lemmas 2 and 3 below.

2 See $\$ 3$ of this paper. 
Definition 1. The set of universal functions (denoted by $U F$ ) is the intersection of all sets of arithmetical functions which include the set $E F$ of elementary functions and are closed under the operations $U$ and.$-^{3}$ If $F$ is an arithmetical function, the set of successors of $F$ is the intersection of all sets of arithmetical functions which include $\{F\}$ and are closed under the operations $\nabla_{k}$ and $\Delta_{k}$ for $k=0,1, \cdots$.

DEFINITION 2. The set of universal conditional functions (denoted by $U C O N D F$ ) has as elements all finite intersections of unions of finitely many complements of elementary functions and at most one elementary function. The set of conditional functions (denoted by $C O N D F)$ has as elements all successors of universal conditional functions. The set of disjunctive functions (denoted by DISJF) has as elements all successors of those universal functions which are finite unions of elementary functions and complements of elementary functions. The set of positive functions (denoted by $P O S F$ ) has as elements all successors of those universal functions which are finite intersections of finite unions of elementary functions. ${ }^{4}$

Definition 3. A set of algebras $S$ is called: a universal class $^{3}$ if $\mathcal{S}=\mathcal{C} \mathcal{L}(F)$ for some function $F \in U F$; a conditional class if $\mathcal{S}=\mathcal{C} \mathcal{L}(F)$ for some $F \in C O N D F$; a disjunctive class if $\mathcal{S}=\mathcal{C} \mathcal{L}(F)$ for some $F \in D I S J F$; a positive class if $S=\mathcal{C} \mathcal{L}(F)$ for some $F \in P O S F$. The family of all universal classes is denoted by $U C{ }^{3}$ that of all conditional classes by CONDC, that of all disjunctive classes by DISJC, and that of all positive classes by POSC.

Definition 4. If, for each $i$ in an index set $I, \mathfrak{A}_{i}=\left\langle A_{i},+\right\rangle$ is an algebra, the direct union of the algebras $\mathfrak{A}_{i}$, denoted by $\mathfrak{u A}_{i}[i \in I]$, is the algebra $\mathfrak{A}=\langle A,+\rangle$ of all functions $u$ on $I$ such that $u(i) \in A_{i}$ for all $i \in I$, and such that $+(u, v, w)$ holds in $\mathfrak{A}$ if and only if, for each $i,+(u(i), v(i), w(i))$ holds in $\mathfrak{A}_{i}$. (If the $\mathfrak{A}_{i}$ are merely sets and not algebras, $\mathfrak{H A}_{i}[i \in I]$ is merely the set of functions defined above.) If $\mathfrak{U}=\langle A,+\rangle=\mathfrak{u}_{i}[i \in I], x$ is an element of $A^{\omega}$ (i.e., a,sequence of elements of $A$ ), and $i \in I$, we denote the sequence $\left\{x_{0}(i), x_{1}(i), \cdots\right\}$ by $\langle x(i)\rangle \cdot(x$ is determined if $\langle x(i)\rangle$ is given for each $i$.)

REMARK. We therefore have for every direct union $\mathfrak{U}=\langle A,+\rangle$ $=\mathfrak{u} \mathfrak{A}_{i}[i \in I]:$ If $F$ is an elementary function, then $F(\mathfrak{U})=\{x \mid x$ $\left.\in A^{\omega} \wedge(\wedge i \in I)\left\{\langle x(i)\rangle \in F\left(\mathfrak{A}_{i}\right)\right\}\right\}$. Dually, if $F$ is the complement of an elementary function, then $F(\mathfrak{A})=\left\{x \mid x \in A^{\omega} \wedge(\vee i \in I)\{\langle x(i)\rangle\right.$ $\left.\left.\in F\left(\mathfrak{A}_{i}\right)\right\}\right\}$.

Definition 5. Let $F, G \in A F$. Let $F=H_{0}, H_{1}, \cdots, H_{n}=G$ be a

${ }^{3}$ Cf. [5].

4 Cf. $[4$, Theorem 11$]$. 
sequence of arithmetical functions for which there exists a set $S$ of functions $f$ from $A F$ to $A F$, together with a sequence of natural numbers $k(1), \cdots, k(n)$, such that for each positive integer $p$ with $p \leqq n$, there occurs at least one of the following cases:

Case 1. $\left(H_{p}=\nabla_{k(p)} H_{p-1}\right) \wedge(\wedge f \in S)\left\{f\left[H_{p}\right]=\nabla_{k(p)} f\left[H_{p-1}\right]\right\}$.

Case 2. $\left(H_{p}=\triangle_{k(p)} H_{p-1}\right) \wedge(\wedge f \in S)\left\{f\left[H_{p}\right]=\Delta_{k(p)} f\left[H_{p-1}\right]\right\}$.

Then the sequence $H_{0}, H_{1}, \cdots, H_{n}$ is called a step sequence from $F$ to $G$, and the set $S$ of functions is called concurrent with the step sequence. (For example, if $f$ is the identity function from $A F$ to $A F$, the set containing $f$ as the sole member is concurrent with all step sequences.)

\section{Sufficient conditions for nonclosure under direct union.}

Lemma 1. Let $F, G \in A F$, and let $G$ be a successor of $F$. Let $H_{0}$, $H_{1}, \cdots, H_{n}$ be a step sequence from $F$ to $G$, and let $I$ be a set of indices for functions $f_{i}$ such that the set $S=\left\{f_{i} \mid i \in I\right\}$ is concurrent with the step sequence. Let $F$ have the following property $P$ : For all direct unions $\mathfrak{A}=\langle A,+\rangle$ of algebras $\mathfrak{A}_{i}$ indexed by $I$, and for all $x \in A^{\omega}$,

$$
(\wedge i \in I)\left\{\langle x(i)\rangle \notin f_{i}[F]\left(\mathfrak{A}_{i}\right)\right\} \rightarrow x \in F(\mathfrak{A}) .
$$

Then each member of the step seguence (hence also $G$ ) has the property $P$.

REMARK. In all applications of this lemma, $I$ will be finite.

Proof. If $\mathfrak{A}=\langle A,+\rangle$ is an algebra, $x \in A^{\omega}$, and $y \in A^{\omega}$ differs from $x$ at most in its $k$ th term, we call $y$ a $k$-sequence for $x$. (For example, if $\mathfrak{A}=\mathfrak{U} \mathfrak{A}_{i}[i \in I]$ and $x \in A^{\omega}$, then $y \in A^{\omega}$ is a $k$-sequence for $x$ if and only if, for each $i$ in $I,\langle y(i)\rangle$ is a $k$-sequence for $\langle x(i)\rangle$.) Suppose that the hypothesis holds; then $H_{0}=F$ has the property $P$. Suppose that $H_{p-1}, p-1<n$, has the property $P$. To prove that $H_{p}$ has the property $P$, suppose that for $\mathfrak{A}$ and $x$ as described,

$$
(\wedge i \in I)\left\{\langle x(i)\rangle \notin f_{i}\left[H_{p}\right]\left(\mathfrak{A}_{i}\right)\right\} .
$$

It remains to show that

$$
x \notin H_{p}(\mathfrak{U}),
$$

and we distinguish the two cases of Definition 5 .

CASE 1. $H_{p}=\nabla_{k(p)} H_{p-1}$, and $f_{i}\left[H_{p}\right]=\nabla_{k(p)} f_{i}\left[H_{p-1}\right]$ for all $f_{i} \in S$. We must show that $x \notin \nabla_{k(p)} H_{p-1}(\mathfrak{A})$, i.e. that no $k(p)$-sequence for $x$ belongs to $H_{p-1}(\mathfrak{A})$. Indeed, let $y \in A^{\omega}$ be a $k(p)$-sequence for $x$. Then, for all $i,\langle y(i)\rangle$ is a $k(p)$-sequence for $x(i)$. But by (2)

$$
(\wedge i \in I)\left\{\langle x(i)\rangle \notin \nabla_{k(p)} f_{i}\left[H_{p-1}\right]\left(\mathfrak{A}_{i}\right)\right\} .
$$

Therefore, for all $i$ : No $k(p)$-sequence for $\langle x(i)\rangle$ belongs to $f_{i}\left[H_{p-1}\right]$ 
$\left(\mathfrak{A}_{i}\right)$, and $\langle y(i)\rangle \notin f_{i}\left[H_{p-1}\right]\left(\mathfrak{A}_{i}\right)$. Hence, by the inductive hypothesis, $y \notin H_{p-1}(\mathfrak{R})$, as was to be shown.

CASE 2. $H_{p}=\triangle_{k(p)} H_{p-1}$, and $f_{i}\left[H_{p}\right]=\triangle_{k(p)} f_{i}\left[H_{p-1}\right]$ for all $f_{i} \in S$. To prove (3) we must show that $x \notin \triangle_{k(p)} H_{p-1}(\mathfrak{H})$, i.e. we must find a $k(p)$-sequence for $x$ which does not belong to $H_{p-1}(\mathfrak{A})$. But by (2),

$$
(\bigwedge i \in I)\left\{\langle x(i)\rangle \in \triangle_{k(p)} f_{i}\left[H_{p-1}\right]\left(\mathfrak{A}_{i}\right)\right\},
$$

i.e., for each $i$ there exists a $k(p)$-sequence for $\langle x(i)\rangle, y_{i}$, such that

$$
y_{i} \notin f_{i}\left[H_{p-1}\right]\left(\mathfrak{A}_{i}\right) .
$$

Choose such a $y_{i}$ for each $i$ and define a $k(p)$-sequence $z$ for $x$ as follows: $z \in A^{\omega} \wedge(\wedge i \in I)\left\{\langle z(i)\rangle=y_{i}\right\}$. By (4) and the inductive hypothesis, $z \notin H_{p-1}(\mathfrak{U})$, as was to be shown.

Lemma 1a. Lemma 1 remains true if (1) is replaced by

$$
(\wedge i \in I)\left\{\langle x(i)\rangle \in f_{i}[F]\left(\mathfrak{A}_{i}\right)\right\} \rightarrow x \in F(\mathfrak{A}) .
$$

Lemma $1 \mathrm{~b}$. Lemma 1 remains true if the reference to the functions $f_{i}$ and to $S$ is deleted and (1) is replaced by

$$
(\wedge i \in I)\left\{\langle x(i)\rangle \in F\left(\mathfrak{A}_{i}\right)\right\} \rightarrow x \in F(\mathfrak{U}) .
$$

Proofs. If $S=\left\{f_{i} \mid i \in I\right\}$ is concurrent with the step sequence $F=H_{0}, H_{1}, \cdots, H_{n}=G$, then any set $S^{\prime}=\left\{g_{i} \mid i \in I\right\}$ in which the $g_{i}$ are functions from $A F$ to $A F$ such that $g_{i}\left[\bar{H}_{p}\right]=f_{i}\left[\overline{H_{p}}\right]$ for all $i \in I$ and $0 \leqq p \leqq n$, is seen to be concurrent with the step sequence $\bar{F}=\bar{H}_{0}, \bar{H}_{1}, \cdots, \bar{H}_{n}=\bar{G}$. Application of Lemma 1 to that step sequence and $S^{\prime}$ gives Lemma $1 \mathrm{a}$.

In particular, in view of the last sentence of Definition 5 , each $f_{i}$ in Lemma 1a may be taken to be the identity function from $A F$ to $A F$; then the result is Lemma $1 b .^{5}$

Lemma 2. Let $F \in U F, G \in A F$, and let $G$ be a successor of $F$. Let

$$
F=\bigcap_{h<l} \bigcup_{i<m(h)} F_{h i},
$$

where $h$ and $i$ run over natural numbers, $l$ is a positive integer, $m$ is a function from $l$ to the positive integers, and for each $F_{h i}$, either $F_{h i} \in E F$ or $\bar{F}_{h i} \in E F$. For each $h<l$, let $m(h)=P(h) \cup Q(h)$, where

$$
P(h)=\left\{i \mid i<m(h) \wedge \bar{F}_{h i} \in E F\right\}, Q(h)=\left\{i \mid i<m(h) \wedge F_{h i} \in E F\right\} .
$$

Let $H_{0}, H_{1}, \cdots, H_{n}$ be a step sequence from $F$ to $G$. Let $S=\left\{f_{h i} \mid h<l\right.$,

- This special case corresponds to a lemma due to Horn [2, Lemma 4], whose method of proof was used for Lemma 1. 
$i<m(h)\}$ be a set of functions concurrent with the step sequence such that

$$
f_{h i}[F]=\underset{r \in P(k) \cup(i)}{\bigcup} F_{h r}
$$

for all $h<l$ and $i<m(h)$. Let $h$ be a natural number less than $l$. Then each member $H_{p}$ of the step sequence (hence also $G$ ) has the following property: For all direct unions $\mathfrak{A}=\langle A,+\rangle$ of algebras $\mathfrak{A}_{i}$ indexed by $m(h)$, and for all $x \in A^{\omega}$

$$
(\bigwedge i<m(h))\left\{\langle x(i)\rangle \notin f_{h i}\left[H_{p}\right]\left(\mathfrak{H}_{i}\right)\right\} \rightarrow x \notin H_{p}(\mathfrak{H}) .
$$

Proof. In view of Lemma 1 , it is sufficient to prove the assertion for $p=0$, i.e. for $H_{0}=F$. Therefore, suppose that $F, G, S, h, \mathfrak{A}$, and $x$ are as in the hypothesis, and that

$$
(\bigwedge i<m(h))\left\{\langle x(i)\rangle \notin \underset{r \in \cup_{P(h) \cup(i)}}{\cup} F_{h r}\left(\mathfrak{A}_{i}\right)\right\} .
$$

It is sufficient to show that

$$
x \notin F(\mathfrak{R}) .
$$

Indeed, by hypothesis, we have $\langle x(i)\rangle \notin F_{h r}\left(\mathfrak{H}_{i}\right)$ for all $i<m(h)$, and all $r \in P(h)$. Hence, by the remark following Definition 4,

$$
(\wedge r \in P(h))\left\{x \in F_{h r}(\mathfrak{R})\right\} .
$$

Also, by hypothesis, $\langle x(i)\rangle \in F_{h i}\left(\mathfrak{A}_{i}\right)$ for all $i \in Q(h)$; hence, by the same remark, $(\wedge i \in Q(h))\left\{x \in F_{h i}(\mathfrak{Q})\right\}$. From this and (7) we conclude that $x \in \cup_{i<m(h)} F_{h i}(\mathfrak{R})$; hence by (5), that $x \notin F(\mathfrak{R})$.

REMARK. We can satisfy the hypothesis of Lemma 2 (and the corresponding part of the hypothesis of Theorem 1 below) for every $G \in A F$. For every such $G$ is a successor of some $F \in U F$ representable in the form (5); ${ }^{4}$ we can then always construct a step sequence from $F$ to $G$ and (by obvious inductive definitions) a set of functions $S$ whose members satisfy (6) and which is concurrent with the step sequence. A similar observation holds for Lemma 3.

TheOREM 1. Let $G \in A F, G \notin C O N D F$, and let $G$ be a successor of $F \in U F$, represented in the form (5) of Lemma 2. Let $H_{0}, H_{1}, \cdots, H_{n}$ be a step sequence from $F$ to $G$, and let the $P(h)$, the $Q(h)$, and the set $S$ be as in Lemma 2. Then, if

$$
(\bigvee h<l)(\wedge i<m(h))\left\{\mathcal{C} \mathcal{L}(G) \nsubseteq \mathcal{C} \mathcal{L}\left(f_{h i}[G]\right)\right\},
$$

$\mathcal{C L}(G)$ is not closed under direct union.

REMARK. The proof will show that the hypothesis $G \notin C O N D F$ is 
unnecessary. In fact, by Theorem 1 and the closure property of conditional classes mentioned in the introduction, or directly by the proof method used for Corollary 2 below, $G \in C O N D F$ is implied by the remaining hypotheses.

Proof. Suppose that the hypothesis holds, and let $h<l$ be such that $(\wedge i<m(h))\left\{\mathcal{C} \mathcal{L}(G) \Phi \mathcal{C} \mathcal{L}\left(f_{h i}[G]\right)\right\}$. For each $i<m(h)$, let $\mathfrak{A}_{i}=\left\langle A_{i},+\right\rangle$ be an algebra such that $\mathfrak{A}_{i} \in \mathcal{C} \mathcal{L}(G), \mathfrak{A}_{i} \notin \mathcal{C} \mathcal{L}\left(f_{h i}[G]\right)$, and let $x_{i} \in A_{i}^{\omega}$ be such that

$$
x_{i} \notin f_{h i}[G]\left(\mathfrak{A}_{i}\right) .
$$

Let $\mathfrak{A}=\langle A,+\rangle=\mathfrak{u A}_{i}[i<m(h)]$, and define $y \in A^{\omega}$ by:

$$
(\wedge i<m(h))\left\{\langle y(i)\rangle=x_{i}\right\} \text {. }
$$

Then, by Lemma 2 and (9), $y \notin G(\mathfrak{U})$. Hence $\mathfrak{A} \notin \mathcal{C} \mathcal{L}(G)$, while for each $i, \mathfrak{A}_{i} \in \mathcal{C} \mathcal{L}(G)$.

CoRollary 1. If $S \in C O N D C$ and $S \in U C, S$ is not closed under direct union.

Proof. By Definition 3, there exists $F \in U F$ such that $S=C \mathcal{L}(F)$; we have $F \notin C O N D F$. Let $F$ be represented in the form (5) of Lemma 2. Consider the step sequence $F=H_{0}=G$, and let the $P(h)$, the $Q(h)$, and the set $S$ be as in Lemma 2. It is sufficient to show that (8) holds with $F$ substituted for $G$. Indeed, if this were not true then

$$
(\wedge h<l)(\vee i<m(h))\left\{\mathcal{C} \mathcal{L}(F) \subseteq \mathcal{C} \mathcal{L}\left(f_{h i}[F]\right)\right\} .
$$

For each $h<l$, choose an $i(h)<m(h)$ such that $\mathcal{L}(F) \subseteq \mathcal{C} \mathcal{L}\left(f_{h, i(h)}[F]\right)$. Then $\mathcal{C} \mathcal{L}(F) \subseteq \mathcal{C} \mathcal{L}\left(\bigcap_{h<l} f_{h, i(h)}[F]\right), \bigcap_{h<l} \quad \mathcal{C} \mathcal{L}\left(G_{h}\right)$ being equal to $\mathcal{C} \mathcal{L}\left(\cap_{h<l} G_{h}\right)$ whenever the $G_{h}, h<l$ are arithmetical functions. But

$$
\bigcap_{h<l} f_{h, i(h)}[F]=\bigcap_{h<l} \bigcup_{r \in P(h) \cup\{i(h)\}} F_{h r} \subseteq F,
$$

and therefore $\mathcal{C} \mathcal{L}\left(\bigcap_{h<l} f_{h, i(h)}[F]\right) \subseteq \mathcal{C} \mathcal{L}(F)$ also, whence $\mathcal{S}=\mathcal{C} \mathcal{L}(F)$ $=\mathcal{C} \mathcal{L}\left(\bigcap_{h<l} f_{h, i(h)}[F]\right)$. The last-named function is a conditional function, and so $S \in C O N D C$, contradicting the hypothesis. Hence (8) must hold as asserted.

Corollary 2. If $\mathcal{Q} \notin C O N D C$ and $S \in D I S J C$, then $S$ is not closed under direct union.

Proof. There exists $G \in D I S J F$ such that $S=\mathcal{C} \mathcal{L}(G)$; we have $G \notin C O N D F$. There exists an $F$ belonging to $U F$ and to $D I S J F$ such that $G$ is a successor of $F$. $F$ may be represented in the form (5), as a union with $l=1$. Let $H_{0}, H_{1}, \cdots, H_{n}$ be a step sequence from $F$ to $G$, 
and let the $P(h)$, the $Q(h)$, and the set $S$ be as in Lemma 2. It is sufficient to show that (8) holds. To prove this, we show first that for every non-negative $p \leqq n$

$$
(\wedge i<m(0))\left\{f_{0 i}\left[H_{p}\right] \subseteq H_{p}\right\} .
$$

Indeed, for $p=0$ we have by $(6):(\wedge i<m(0))\left\{f_{0 i}[F] \subseteq F\right\}$. If $(10)$ is true with $p-1<n$ substituted for $p$, then, for all $i<m(0)$ and for $k=0,1, \cdots$, both $\nabla_{k} f_{0 i}\left[H_{p-1}\right] \subseteq \nabla_{k} H_{p-1}$ and $\triangle_{k} f_{0 i}\left[H_{p-1}\right] \subseteq \triangle_{k} H_{p-1}$; and then (10) holds both in Case 1 and in Case 2 of Definition 5.

Now, if (8) did not hold, we should have

$$
(\bigvee i<m(0))\left\{\subset \mathcal{L}(G) \subseteq \mathcal{C} \mathcal{L}\left(f_{0 i}[G]\right)\right\} .
$$

But by (10), with $p=n, H_{n}=G,(\wedge i<m(0))\left\{\mathcal{C} \mathcal{L}\left(f_{0 i}[G]\right) \subseteq \mathcal{C} \mathcal{L}(G)\right\}$, and hence, for some $i<m(0), \mathcal{S}=\mathcal{C} \mathcal{L}(G)=\mathcal{C} \mathcal{L}\left(f_{0 i}[G]\right)$. Since $f_{0 i}[G]$ $\in C O N D F$, we find $\delta \in C O N D C$, contradicting the hypothesis. Hence (8) must hold.

Lemma 3. Let $G \in P O S F$. Let $G$ be a successor of the function $F$ belonging to $U F$ and to POSF and represented in the form (5) of Lemma 2, $H_{0}, H_{1}, \cdots, H_{n}$ be a step sequence from $F$ to $G$, and let the $P(h)$ and $Q(h)$ be as in Lemma 2; we may assume all the $P(h)$ to be empty. Let $J=\mathfrak{H} Q(h)[h<l]$, and let $S=\left\{f_{j} \mid j \in J\right\}$ be a set of functions concurrent with the step sequence such that

$$
f_{j}[F]=\bigcap_{h<l} F_{h, j(h)}
$$

for all $j \in J$. Then each member $H_{p}$ of the step sequence (hence also $G$ ) has the following property: For all direct unions $\mathfrak{A}=\langle A,+\rangle$ of algebras $\mathfrak{A}_{j}$ indexed by $J$, and for all $x \in A^{\omega}$

$$
(\wedge j \in J)\left\{\langle x(j)\rangle \notin f_{j}\left[H_{p}\right]\left(\mathfrak{A}_{j}\right)\right\} \rightarrow x \in H_{p}(\mathfrak{A}) .
$$

Proof. In view of Lemma 1 , it is sufficient to prove the assertion for $p=0$, i.e. for $H_{0}=F$. Suppose that $F, G, S, \mathfrak{A}$, and $x$ are as in the hypothesis, and that

$$
(\wedge j \in J)\left\{\langle x(j)\rangle \notin \bigcap_{h<l} F_{h, j(h)}\left(\mathfrak{A}_{j}\right)\right\} .
$$

It is sufficient to show that $x \in F(\mathfrak{H})$. Indeed, by hypothesis, $(\wedge j \in J)$ $(\bigvee h<l)\left\{\langle x(j)\rangle \notin F_{h, j(h)}\left(\mathfrak{A}_{j}\right)\right\}$. Since $F_{h, j(h)} \in E F$, we conclude that $(\wedge j \in J)(\bigvee h<l)\left\{x \in F_{h, j(h)}(\mathfrak{A})\right\}$, and

$$
(\wedge j \in J)\left\{x \in \bigcap_{h<l} F_{h, j(h)}(\mathfrak{A})\right\} .
$$


Now, if we had $x \in F(\mathfrak{A})$, then, by (5) and the emptiness of the $P(h),(\wedge h<l)(\bigvee i \in Q(h))\left\{x \in F_{h i}(\mathfrak{Q})\right\}$. We form an element $j$ of $J$ by taking, for each $h<l, j(h) \in Q(h)$ to be such that $x \in F_{h, j(h)}(\mathfrak{H})$. Then $x \in \bigcap_{h<l} F_{h, j(h)}(\mathfrak{A})$, contradicting (12). Hence $x \notin F(\mathfrak{A})$.

THEOREM 2. If $\mathcal{C} \in C O N D C, S \in P O S C$, then $S$ is not closed under direct union.

Proof. There exist $G$ and $F$ as in Lemma 3 such that $\mathcal{S}=\mathcal{C} \mathcal{L}(G)$; we have $G \oplus C O N D F$. Let $H_{0}, H_{1}, \cdots, H_{n}$ be a step sequence from $F$ to $G$, and let the $P(h)$ and $Q(h), J$ and $S$ be as in Lemma 3. In view of Lemma 3 , it will be sufficient to find, for each $j \in J$, an algebra $\mathfrak{A}_{j}=\left\langle A_{j},+\right\rangle$ such that $\mathfrak{A}_{j} \in \mathcal{S}, \mathfrak{A}_{j} \in \mathcal{C} \mathcal{L}\left(f_{j}[G]\right)$. For then we may choose, for each $j, x_{j} \in A_{j}^{\omega}$ such that $x_{j} \notin f_{j}[G]\left(\mathfrak{A}_{j}\right)$, and, if $\mathfrak{A}=\langle A,+\rangle$ $=\mathfrak{u} \mathfrak{A}_{j}[j \in J]$, we define $y \in A^{\omega}$ by letting $\langle y(j)\rangle=x_{j}$ for each $j \in J$. Then, by Lemma $3, y \in G(\mathfrak{H})$, and $\mathfrak{A} \in \mathcal{C} \mathcal{L}(G)=\mathcal{S}$, while $\mathfrak{A}_{j} \in \mathcal{S}$ for each $j \in J$, which will prove the theorem.

It remains to show that for each $j \in J$, such an $\mathfrak{A}_{j}$ exists. Indeed, if not, we have

$$
(\vee j \in J)\left\{\delta \subseteq \mathcal{C} \mathcal{L}\left(f_{j}[G]\right)\right\} .
$$

On the other hand, by (11), $(\wedge j \in J)\left\{f_{j}[F] \subseteq F\right\}$, and this implies, as in the proof of Corollary $2,(\wedge j \in J)\left\{f_{j}[G] \subseteq G\right\}$. Therefore $(\wedge j \in J)$ $\left\{\mathcal{L}\left(f_{j}[G]\right) \subseteq \mathcal{C} \mathcal{L}(G)=\mathcal{S}\right\}$, which, with (13), implies $(\vee j \in J)$ $\left\{S=\mathcal{C} \mathcal{L}\left(f_{j}[G]\right)\right\}$. But, in view of (11), $f_{j}[G] \in C O N D F$, and so $\delta \in \operatorname{CONDC}$, contradicting the hypothesis. It follows that for each $j \in J$, an $\mathfrak{A}_{j}$ as described exists.

3. A sufficient condition for closure under direct union. As was mentioned in the introduction, all conditional classes of algebras are known to be closed under direct union. (The proof of this fact is like Horn's corresponding proof [2, Theorems 3 and 4]: One shows that (1b) holds if $F \in U C O N D F$, and the assertion follows by use of Lemma 1b). Another result due to Horn [2, Theorem 10], which carries over into the theory of arithmetical classes, implies that every subfamily of $A C$, disjoint with $C O N D C$, which can be described in terms of the structure of the canonical representations ${ }^{4}$ of the arithmetical functions characterizing the family members, has at least one member class which is not closed under direct union. We conclude from the corollaries and from Theorem 2 of the preceding section that in some subfamilies of this kind (namely, the subfamilies of UC, DISJC, and POSC consisting of the respective members not in CONDC) all members fail to be closed under direct union. But Theorem 3 of this section makes it seem unlikely that the same 
should be true for the entire subfamily of $A C$ consisting of the members not in CONDC; for this theorem gives a sufficient condition for closure of which there seems no reason to assume that it applies to conditional classes only. There remains the problem of finding a condition both sufficient and necessary for closure, which would clarify the status of those nonconditional arithmetical classes satisfying none of the conditions mentioned in Theorems 1, 2, and 3 .

Theorem 3. Let $G \in A F$, and let $G$ be a successor of $F \in U F$, represented in the form (5). Let $H_{0}, H_{1}, \cdots, H_{n}$ be a step sequence from $F$ to $G$, and let the $P(h)$ and $Q(h)$ be as in Lemma 2. Let there exist a set $S=\left\{\phi_{0}, \phi_{1}\right\}$ of functions concurrent with the step sequence, a decomposition of $l$ into disjoint sets $L(0), L(1), L(2)$, one set $R(h)$ for each $h<l$, and one number $r(h) \in Q(h)$ for each $h \in L(2)$ such that

$$
\begin{aligned}
& L(0) \cup L(1) \subseteq\{h \mid h<l \wedge P(h) \neq \Lambda\}, \\
& L(2) \subseteq\{h \mid h<l \wedge Q(h) \neq \Lambda\} \text {; } \\
& R(h)= \begin{cases}P(h) & \text { if } h \in L(0) \cup L(1), \\
\{r(h)\} & \text { if } h \in L(2) ;\end{cases} \\
& \phi_{0}[F]=\bigcap_{h \in L(0) \cup L(2)} \bigcup_{r \in R(h)}^{U} F_{h r}, \\
& \phi_{1}[F]=\bigcap_{h \in L(1) \cup L(2)} \bigcup_{r \in R(h)} F_{h r} ; \\
& \mathcal{L} \mathcal{L}(G) \subseteq \mathcal{C} \mathcal{L}\left(\phi_{0}[G]\right) \cap \mathcal{C} \mathcal{L}\left(\phi_{1}[G]\right) .
\end{aligned}
$$

Then $\mathcal{C} \mathcal{L}(G)$ is closed under direct union.

REMARK. $\phi_{0}\left[H_{p}\right]$ and $\phi_{1}\left[H_{p}\right]$ belong to CONDF for each $H_{p}$ of the sequence. If $L(0)$ is empty, then $L(1) \cup L(2)=l$. One then sees as in the proof of Corollary 2 that $\mathcal{C} \mathcal{L}\left(\phi_{1}[G]\right) \subseteq \mathcal{C} \mathcal{L}(G)$, and by (17) $\mathcal{L}(G)=\mathcal{C} \mathcal{L}\left(\phi_{1}[G]\right) \in C O N D C$, so that the assertion follows from the known closure property of conditional classes. The same is true if $L(1)$ is empty. The proof is not affected if $L(2)$ is empty.

Proof. Suppose the hypothesis holds. By the preceding remark, we may assume that,neither $L(0)$ nor $L(1)$ is empty.

Let $I$ be an index set which has at least two elements. Let $i_{0}$ be a fixed element of $I$. We define a set $S^{\prime}=\left\{f_{i} \mid i \in I\right\}$ of functions concurrent with the given step sequence as follows:

$$
f_{i}=\left\{\begin{array}{l}
\phi_{0} \text { for } i=i_{0}, \\
\phi_{1} \text { for } i \neq i_{0} .
\end{array}\right.
$$

We shall prove that $G$ has the following property $P^{\prime}$ : For all direct 
unions $\mathfrak{A}=\langle A,+\rangle$ of algebras $\mathfrak{A}_{i}$ indexed by $I$, and for all $x \in A^{\omega}$,

$$
(\wedge i \in I)\left\{\langle x(i)\rangle \in f_{i}[G]\left(\mathfrak{A}_{i}\right)\right\} \rightarrow x \in G(\mathfrak{A}) .
$$

Indeed, by Lemma $1 \mathrm{a}$ it is sufficient to prove that $F$ has the property $P^{\prime}$. To show this, suppose that for $\mathfrak{A}$ and $x$ as described, the antecedent of (19) holds with $F$ substituted for $G$. Then by (18), (16), and (15)

$$
\left\langle x\left(i_{0}\right)\right\rangle \in\left[\bigcap_{h \text { in } L(0)} \underset{r \text { in } P(h)}{\bigcup} F_{h r}\left(\mathfrak{A}_{i_{0}}\right)\right] \cap\left[\bigcap_{h \text { in } L(2)} F_{h, r(h)}\left(\mathfrak{A}_{i_{0}}\right)\right],
$$

and

$$
\begin{aligned}
\left(\wedge i \in I-\left\{i_{0}\right\}\right)\{\langle x(i)\rangle & \in\left[\bigcap_{h \operatorname{in} L(1)} \underset{r \text { in } P(h)}{\cup} F_{h r}\left(\mathfrak{A}_{i}\right)\right] \\
& \left.\cap\left[\bigcap_{h \operatorname{in} L(2)} F_{h, r(h)}\left(\mathfrak{A}_{i}\right)\right]\right\} .
\end{aligned}
$$

Therefore,

$$
\begin{aligned}
& (\wedge h \in L(0))(\vee r \in P(h))\left\{\left\langle x\left(i_{0}\right)\right\rangle \in F_{h r}\left(\mathfrak{A}_{i_{0}}\right)\right\}, \\
& \left(\wedge i \in I-\left\{i_{0}\right\}\right)(\wedge h \in L(1))(\bigvee r \in P(h))\left\{\langle x(i)\rangle \in F_{h r}\left(\mathfrak{A}_{i}\right)\right\},
\end{aligned}
$$

and

$$
\left(\bigwedge_{i} \in I\right)(\wedge h \in L(2))\left\{\langle x(i)\rangle \in F_{h, r(h)}\left(\mathfrak{A}_{i}\right)\right\} .
$$

The $F_{h r}$ in the first two statements are complements of elementary functions, and the $F_{h, r(h)}$ in the third statement are elementary functions. Hence, by the remark following Definition 4,

$$
(\wedge h \in L(0) \cup L(1))\left(\vee_{r} \in P(h)\right)\left\{x \in F_{h r}(\mathfrak{A})\right\},
$$

and

$$
(\wedge h \in L(2))\left\{x \in F_{h, r(h)}(\mathfrak{X})\right\} .
$$

Therefore $x \in \bigcap_{h<l} \bigcup_{r<m(h)} F_{h r}(\mathfrak{H})=F(\mathfrak{H})$, and $F$, hence also $G$, has the property $P^{\prime}$ as asserted.

To prove the theorem, let $\mathfrak{A}=\langle A,+\rangle$ be a direct union of algebras $\mathfrak{A}_{i}=\left\langle A_{i},+\right\rangle$ indexed by a set $I$, such that $\mathfrak{A}_{i} \in \mathcal{C} \mathcal{L}(G)$ for all $i \in I$. We have to show that $\mathfrak{A} \in \mathcal{C} \mathcal{L}(G)$, i.e., that for all $x \in A^{\omega}$

$$
x \in G(\mathfrak{A}) \text {. }
$$

We may assume that $I$ has at least two elements. We define the set $S^{\prime}=\left\{f_{i} \mid i \in I\right\}$ as in the first part of the proof. Then, by (17), $\mathcal{C} \mathcal{L}(G)$ 
$\subseteq \mathcal{C} \mathcal{L}\left(f_{i}[G]\right)$ for all $i \in I$. Hence for all $i \in I, \mathfrak{A}_{i} \in \mathcal{C} \mathcal{L}\left(f_{i}[G]\right)$. Let $x \in A^{\omega}$, then for all $i \in I,\langle x(i)\rangle \in A_{i}^{\omega}$, and therefore $(\wedge i \in I)\{\langle x(i)\rangle$ $\left.\in f_{i}[G]\left(\mathfrak{A}_{i}\right)\right\}$. Since $G$ has the property $P^{\prime}$ corresponding to $I$ and $S^{\prime}$, (20) now follows from (19). This proves Theorem 3.

REMARK. If we change the hypothesis of Theorem 3 by replacing the positive integer 2 by the positive integer $s, 2 \leqq s \leqq l$ (i.e., replacing the union of $L(0)$ and $L(1)$ by the union of $s$ disjoint nonempty sets $L(0), L(1), \cdots, L(s-1)$, replacing $L(2)$ by the possibly empty set $L(s)$, introducing $s$ concurrent functions $\phi_{0}, \phi_{1}, \cdots, \phi_{s-1}$ such that $\phi_{t}[F]=\bigcap_{h \in L(t) \cup L(s)} \bigcup_{r \in R(h)} F_{h r}$ for each $t<s$, and replacing (17) by: $\left.\mathcal{L}(G) \subseteq n_{t<s} \mathcal{C} \mathcal{L}\left(\phi_{t}[G]\right)\right)$, we can prove by the same method that $\mathcal{L}(G)$ is closed under passage to direct unions of at least $s$ algebras.

\section{REFERENCES}

1. J. C. C. McKinsey, The decision problem for some classes of sentences without quantifiers, J. Symbolic Logic vol. 8 (1943) pp. 61-76.

2. Alfred Horn, On sentences which are true of direct unions of algebras, J. Symbolic Logic vol. 16 (1951) pp. 14-21.

3. R. C. Lyndon, Review of 2, J. Symbolic Logic vol. 16 (1951) pp. 216-217.

4. Alfred Tarski, Some notions and methods on the borderline of algebra and metamathematics, Proceedings of the International Congress of Mathematicians, Cambridge, 1950, Providence, 1952, pp. 705-720.

5. - Universal arithmetical classes of mathematical systems, Bull. Amer. Math. Soc. Abstract 59-4-500.

Rensselaer Polytechnic Institute 